\title{
BMJ Open Pragmatic trial evaluating the effectiveness of a patient navigator to decrease emergency room utilisation in transition age youth with chronic conditions: the Transition Navigator Trial protocol
}

\author{
Susan Samuel (D) , ${ }^{1}$ Gina Dimitropoulos, ${ }^{2}$ Kyleigh Schraeder (D) , ${ }^{3}$ \\ Scott Klarenbach, ${ }^{4}$ Alberto Nettel-Aguirre ${ }^{5}$ Greg Guilcher, ${ }^{6}$ Daniele Pacaud, ${ }^{7}$ \\ Jorge Pinzon, ${ }^{8}$ Eddy Lang, ${ }^{9}$ Gail Andrew, ${ }^{10}$ Lonnie Zwaigenbaum, ${ }^{11}$ \\ Shannon Scott, ${ }^{12}$ Kerry McBrien, ${ }^{13}$ Lorraine Hamiwka, ${ }^{14}$ Andrew Mackie ${ }^{15}$
}

To cite: Samuel S, Dimitropoulos G, Schraeder K, et al. Pragmatic trial evaluating the effectiveness of a patient navigator to decrease emergency room utilisation in transition age youth with chronic conditions: the Transition Navigator Trial protocol. BMJ Open 2019;9:e034309. doi:10.1136/ bmjopen-2019-034309

- Prepublication history and additional material for this paper are available online. To view these files, please visit the journal online (http://dx.doi org/10.1136/bmjopen-2019034309).

Received 13 September 2019 Revised 25 October 2019 Accepted 31 October 2019

Check for updates

(c) Author(s) (or their employer(s)) 2019. Re-use permitted under CC BY-NC. No commercial re-use. See rights and permissions. Published by BMJ.

For numbered affiliations see end of article.

Correspondence to

Dr Susan Samuel;

susan.samue|@ahs.ca

\section{ABSTRACT}

Introduction Transition to adult care is a challenging and complex process for youth with special healthcare needs. We aim to compare effectiveness of a patient navigator service in reducing emergency room (ER) use among adolescents with chronic health conditions transitioning to adult care.

Methods and analysis Pragmatic randomised controlled trial parallel group design comparing ER visit rates between patients with access to a personalised navigator intervention compared with usual care. Unit of randomisation is the patient. Treatment assignment will not be blinded. Embedded qualitative study to understand navigator's role and cost analysis attributable to the intervention will be performed. Patients aged 16-21 years, followed within a chronic disease clinic, expected to be transferred to adult care within 12 months and residing in Alberta during study period will be recruited from three tertiary care paediatric hospitals. Sample size will be 300 in each arm. Navigator intervention over 24 months is designed to assist participants in four domains: transition preparation, health system brokering, socioeconomic determinants of health and self-management. Primary outcome is ER visit rate during observation period. Secondary outcomes are ambulatory and inpatient care utilisation measures, as well as Transition Readiness Assessment Questionnaire score, and Short-Form Health Survey 12 (SF-12) score at 6 and 18 months postrandomisation. Poisson regression will compare rates of ER/urgent care visits between navigator and control participants, using intention to treat principle. Cost analysis of the intervention will be conducted. Thematic analysis will be used to identify perceptions of stakeholders regarding the role of navigators.

Ethics and dissemination Ethics approval was obtained from the University of Calgary Conjoint Health Research Ethics Board (REB \#162561) and the University of Alberta Health Research Ethics Board (Pro00077325). Our team is composed of diverse stakeholders who are committed
Strengths and limitations of this study

Population-based sample from one Canadian province with universal health coverage.

- Pragmatic randomised controlled trial design with broad inclusion criteria, and with an intervention embedded in a real world healthcare setting.

- Participants are not blinded to the treatment arms, but blinded to primary outcome.

- Contamination may occur from clinic-based interventions which may duplicate some of the services the patient navigator may provide.

- Participant recruitment to achieve the prespecified sample size is anticipated to be a challenge; particular attention to youth engagement strategies is required.

to improving transition of care who will assist with dissemination of results.

Trial registration number NCT03342495.

\section{INTRODUCTION}

Approximately $15 \%-20 \%$ of adolescents in North America live with a chronic health condition, defined as a condition that lasts at least 3 months, is not yet curable, affects a child's normal activities and requires ongoing care. ${ }^{1}$ The majority $(>90 \%)$ will require transfer from paediatric to adult services. ${ }^{12}$ Suboptimal transition to adult care leads to poor adherence with ambulatory care management, health deterioration and increased use of costly emergent health services. ${ }^{3} 4$ Patient navigators are a promising, but unproven intervention to facilitate planned transitions from paediatric to adult 
care, and improve patient experience and outcomes. ${ }^{5}$ Published studies describing patient navigator services are mostly single-centre and single-disease cohort studies, with non-randomised designs, thus, limiting generalisability to other health jurisdictions and disease populations. ${ }^{5}$ Further, interventions requiring highly skilled healthcare workers tend to be expensive, and to justify such an intervention, a cost evaluation is necessary. To address these challenges, we designed a pragmatic randomised controlled trial, the Transition Navigator Trial (TNT), the protocol for which is described in this paper.

\section{Trial objectives}

The primary objective is to evaluate the impact of a personalised transition to adult care intervention (access to a patient navigator) compared with usual care for individuals aged 16-21 years living with chronic health conditions who are transferring to adult care with respect to: (a) emergency room (ER)/urgent care visits (primary outcome); and (b) inpatient and ambulatory care utilisation, transition readiness scores and patient-reported health status (secondary outcomes). Secondary objectives are: (a) to determine the net healthcare cost impact attributable to the patient navigator intervention; and (b) to obtain perceptions of stakeholders regarding the role of patient navigators in reducing barriers to adultoriented ambulatory care.

\section{Hypotheses}

The patient navigator intervention will reduce all-cause ER/urgent care visit rates, improve transition readiness scores and patient-reported health status, and generate cost savings for the health system.

\section{METHODS AND ANALYSIS}

This study will be conducted in accordance with the Standard Protocol Items: Recommendations for Interventional Trials checklist ${ }^{6-8}$ and Consolidated Standards of Reporting Trials statement on pragmatic trial extension ${ }^{9}$ (RCT), and COREQ. ${ }^{1011}$

\section{Study design and setting}

This study will use a parallel group, pragmatic RCT ${ }^{9}$ design (figures 1 and 2) with an embedded qualitative study. The RCT involves random allocation of young adults (ages 16-21 years) with a chronic medical/mental health condition to either a personalised transition intervention (access to a patient navigator) or usual transitional care at one of the three tertiary care paediatric hospitals in Alberta, Canada.

Alberta, with a population of 4.1 million, has a universal publicly funded healthcare system that covers over $99 \%$ of the population. ${ }^{12}$ Patients will be recruited from three tertiary care paediatric hospitals: the Stollery Children's Hospital, Alberta Children's Hospital and Glenrose Rehabilitation Hospital.

\section{Recruitment}

Eligible participants will be identified from 41 paediatric specialty clinics at the three participating hospitals (figure 3). These clinics were selected after extensive stakeholder input, as these patient groups have high potential for adverse outcomes if transitions are not managed optimally. ${ }^{42-15}$ Participants will have chronic health conditions in these broad categories: endocrine, gastrointestinal, neurological, neurodevelopmental, rheumatological, renal, cardiac, haematological, respiratory and metabolic/genetic. The primary caregiver

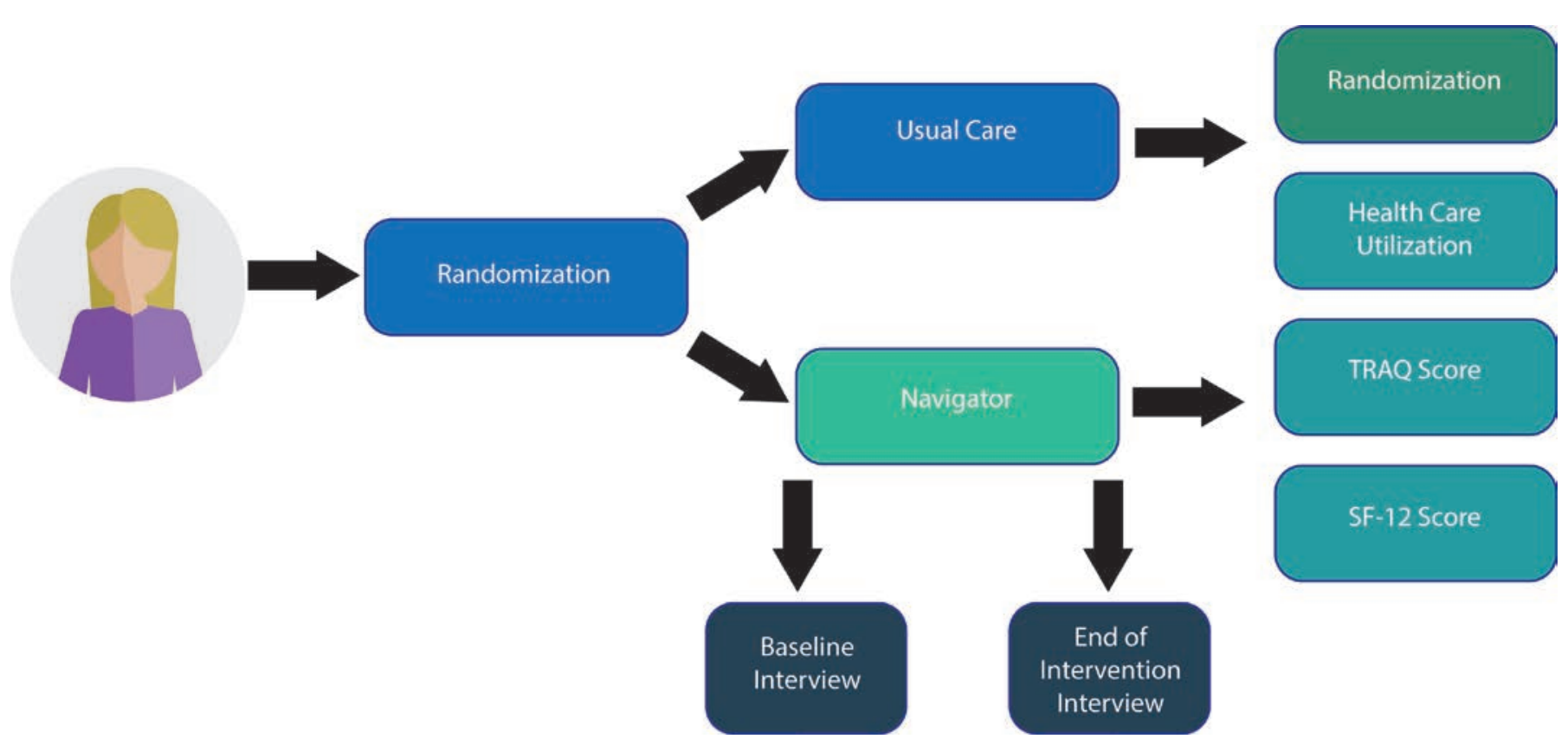

Figure 1 Trial design. A randomised controlled trial. SF-12, Short-Form 12 Health Survey; TRAQ, Transition Readiness Assessment Questionnaire. 


\section{Randomization}

Assessed for eligibility $(n=)$

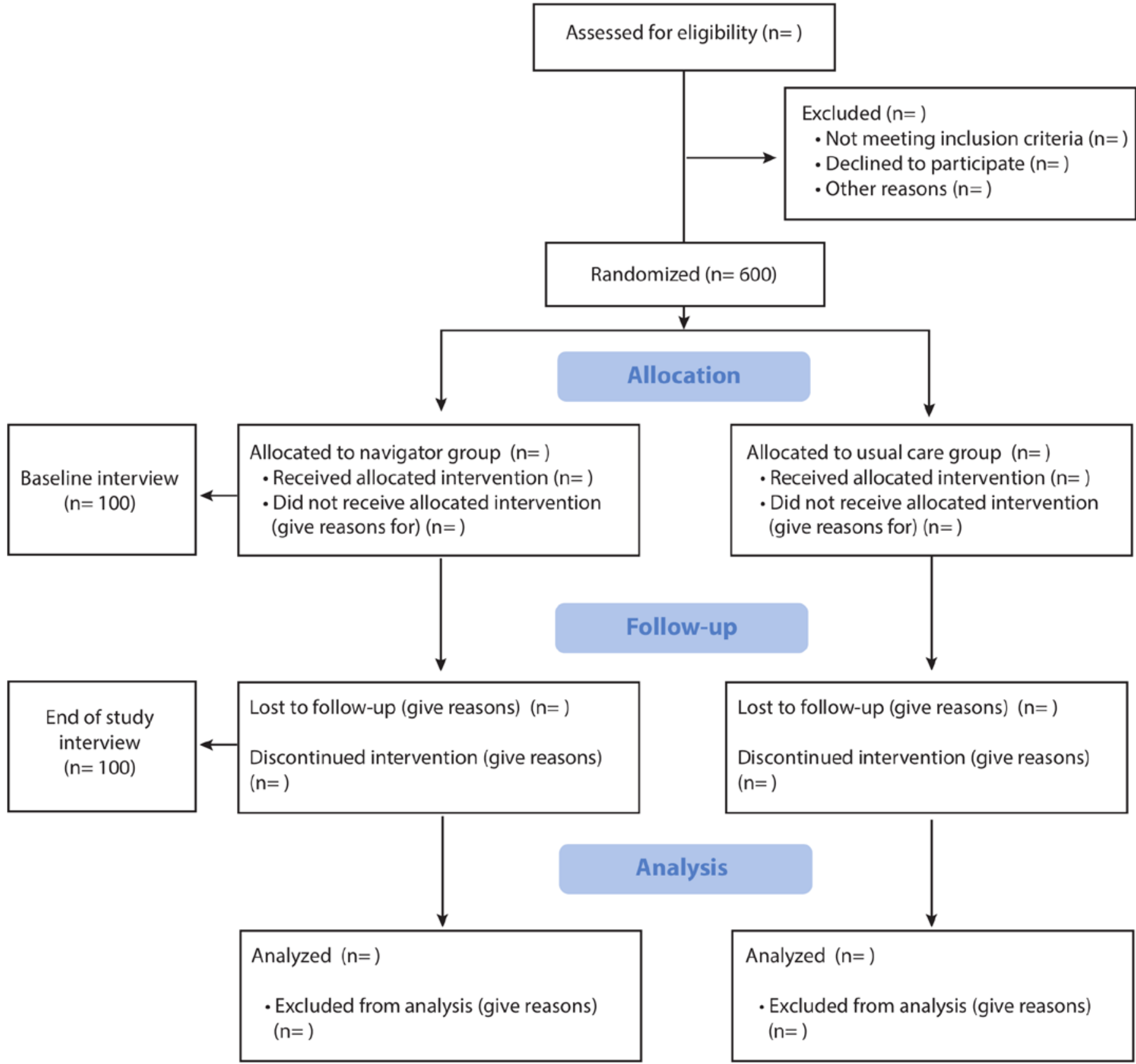

Figure 2 Consolidated Standards of Reporting Trials diagram.

(legal guardian or parent) of the young adult will also be considered a study participant if he/she is willing; however, parent/guardian involvement is not a requirement. Primary caregiver will also provide information required for the study should the patient be non-verbal or lack capacity to participate in the study.

Potentially eligible participants will be recruited through various methods including: (1) clinic staff identifying potential participants and requesting consent to contact by the study team, (2) patients can directly self-refer using a generic study email or phone number provided in recruitment posters and (3) using mail-outs to potentially eligible participants who have used health services at the participating hospitals.

Trained research assistants are responsible for responding to any queries for enrolment via telephone or email. These research assistants are also responsible for screening potential participants for eligibility. The screening process is being conducted in person or by phone.

\section{Inclusion criteria}

To be eligible, participants must: (1) be between 16 and 21 years of age at the time of enrolment, (2) be receiving 


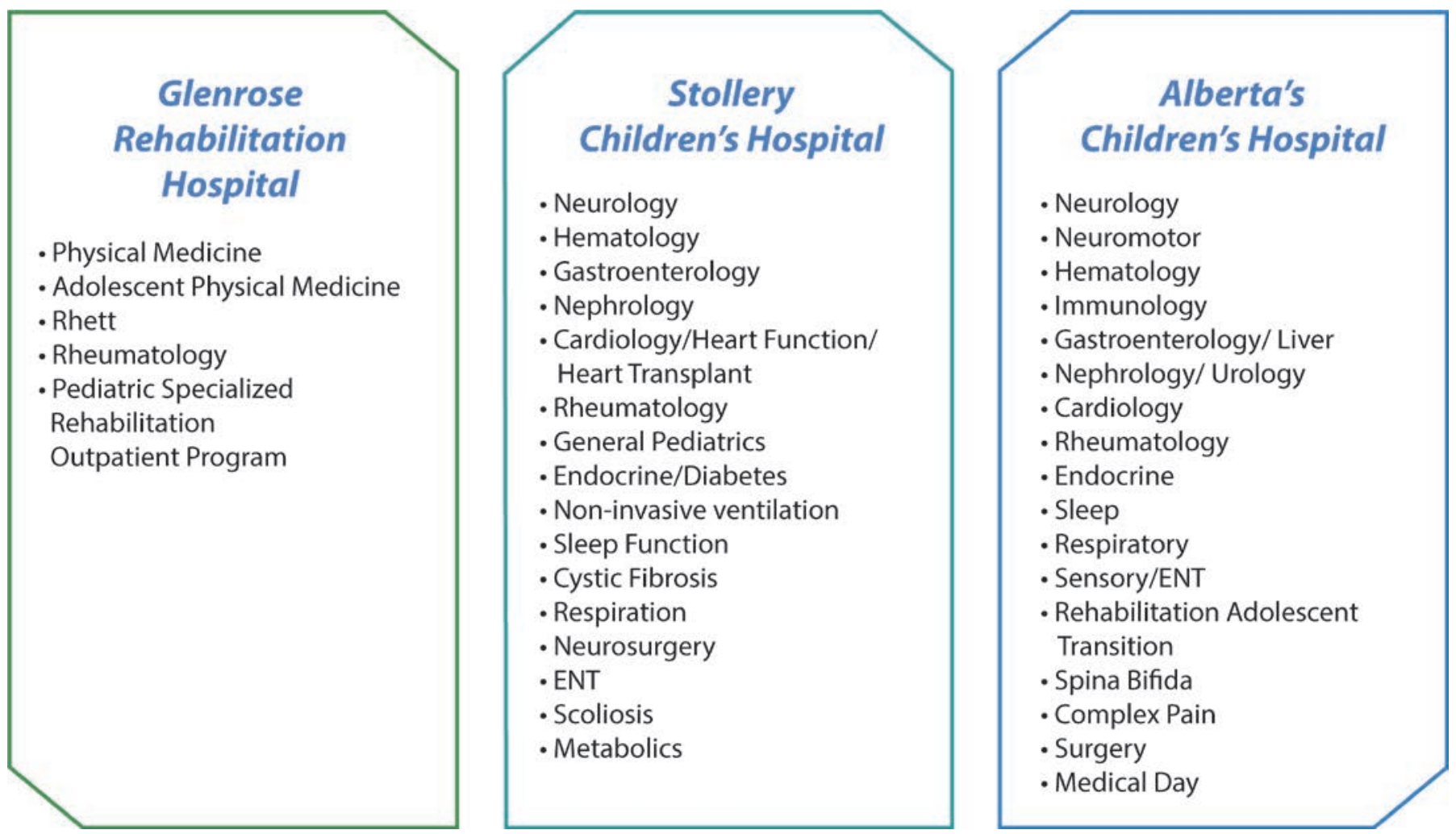

Figure 3 Participating clinics. clinics participating in the Transition Navigator Trial. ENT, ear, nose and throat.

care from at least one of the selected paediatric outpatient hospital and community clinics (figure 3), (3) have a chronic medical condition (defined as conditions which are $>3$ months in duration and/or lifelong with multiple morbidities and/or multi-organ/system manifestations or conditions which typically affect a single organ/ system $)^{1617}$ and (4) be expected to be transferred to adult specialty care in the next 12 months.

\section{Exclusion criteria}

Exclusion criteria will be: (1) enrolled in another transition-related study involving a navigator or similar intervention, (2) transfer will not occur during the time interval for the study, (3) will be moving out of Alberta during the study (eg, going away for college) resulting in inability to report on primary outcome (ER visits) within the province, (4) inability to read and write in English.

\section{Consent}

Informed written consent will be obtained from all participants prior to enrolment (see online supplementary file 1 ). For patients who are minors (age 16-17), informed assent will be obtained where appropriate. When the patient is considered a mature minor (after a capacity assessment by the responsible physician) or at age 18, we will obtain consent. If a patient is consenting for him/ herself, then consent forms will ask participants for permission to contact their parents/guardians as needed to facilitate care, and also for permission to disclose medical information to parents/guardians. Should the participant decline parent involvement in the study, parents will not be contacted nor will health information be provided to the parent.

The primary caregiver will consent for his/her own respective participation. Primary caregivers will also consent on behalf of young adult participants who lack capacity to do so themselves due to developmental delay. Consent for disclosure of personal health numbers (PHN) assigned by Alberta Health for universal healthcare access will be obtained, to allow examination of health service utilisation at the patient level by linkage to administrative health datasets.

Participation will be voluntary and participants will be free to withdraw at any time. A small incentive will be offered to participants ( $\$ 25$ at enrolment and $\$ 25$ at study end), as a token of appreciation of their participation.

\section{Study Timeline}

Participants will be recruited over $\sim 42$ months. Recruitment started in January 2018, and will continue until target enrolment is reached. The duration of navigator support for participants in the intervention arm will be up to 24 months after randomisation, and a minimum of 12 months for those enrolled later in the recruitment period. All participants will be observed for a minimum of 12, and maximum of 42 months. See timeline in figure 4. A schematic diagram outlining the schedule of enrolment, assessments and visits is shown in table 1 .

\section{Feasibility and sample size}

There are approximately 600 prevalent patients between 16 and 18 years of age receiving care at each of the Alberta 
12-18 months 18-24 months 24-30 months 30-36 months 36-42 months $42-48$ months $48-54$ months $54-60$ months

Recruitment and Randomization

Patient Navigator intervention vs treatment as usual

Baseline interviews for intervention group

End of study interviews for intervention group participants

Focus groups with

stakeholders

Data analysis

Figure 4 Study timeline.

share results

Children's Hospital and Stollery Children's Hospital. The Glenrose Rehabilitation Hospital has approximately 250 patients aged 16-18 years. Estimated consent rate is $79 \%$ (based on our experience with transition trials). ${ }^{18} 19$ We expect to recruit approximately 14-15 patients per month to reach our target sample size in 42 months. Clinic 'champions' (physician, nurse or social worker leads) have been identified at all participating clinics to liaise with the study team and facilitate recruitment. A maximum case-load of 140-150 patients per patient navigator (one each in Edmonton and Calgary) is anticipated. This volume is considered feasible based on a similar case load of paediatric clinicians who have provided paediatric to adult transitional care in Alberta.

Table 1 Schedule of assessments for participants in the trial.

Months postrandomisation

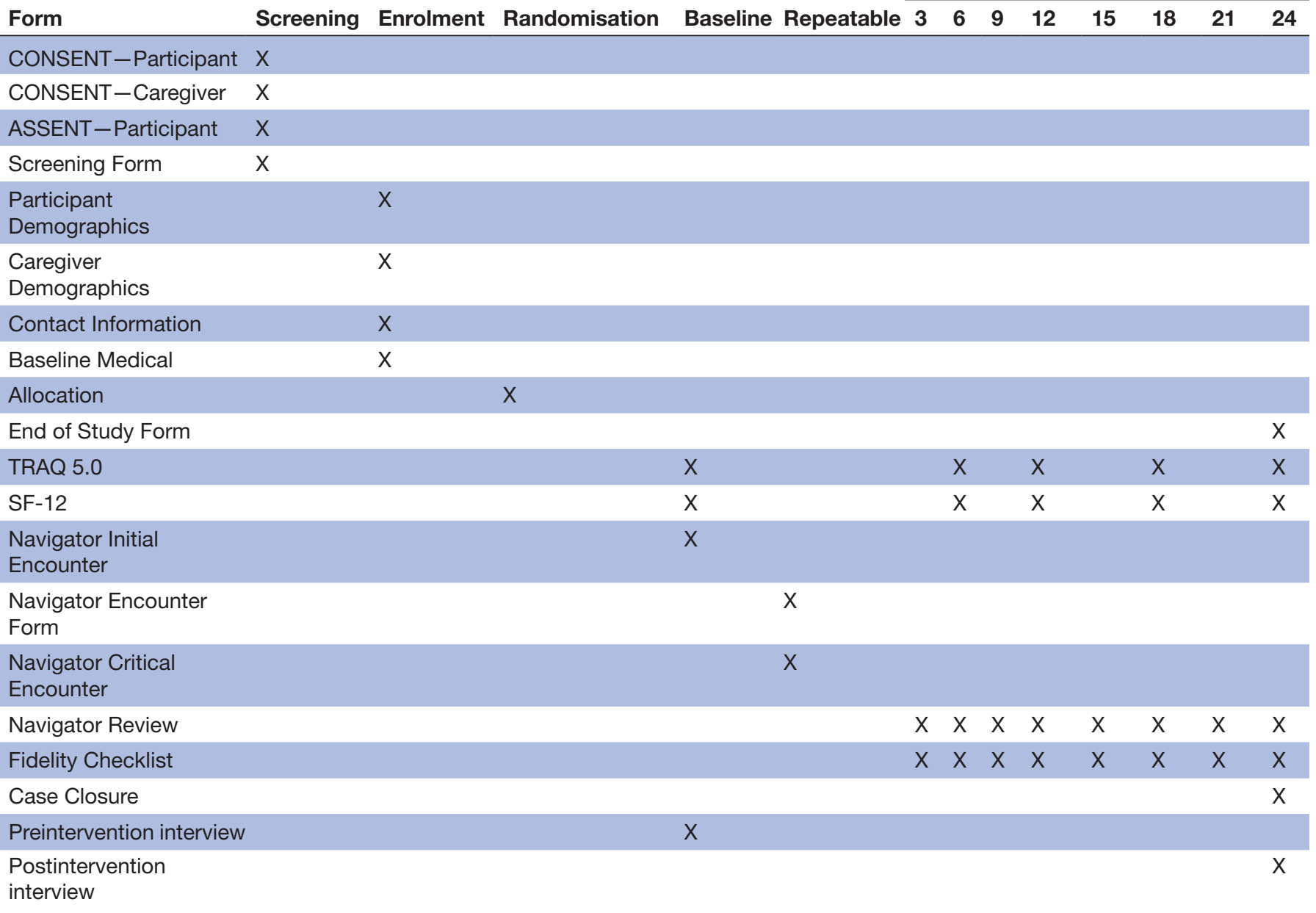

SF-12, Short-Form 12 Health Survey; TRAQ, Transition Readiness Assessment Questionnaire. 
Sample size calculation was based on the primary outcome (ie, ER/urgent care visit rate) during the period of observation. The baseline ER/urgent care visit rate observed within a diverse cohort of transitioning patients in Calgary, as identified using available administrative data, is 51 per 100 person years of follow-up, for age $\geq 18$ years. Our team, composed of stakeholders from various levels of health service delivery, confirmed that a minimum clinically important difference between groups is a 20\% (relative change) drop in ER visits. Based on effect size seen in a prior study evaluating transition navigators' impact on diabetic ketoacidosis admissions in diabetic patients, ${ }^{20}$ we expect a $20 \%-25 \%$ relative rate reduction in the intervention group compared with the control group. Assuming an ER/urgent care visit rate of 40 per 100 person years ( $21 \%$ rate reduction) in the intervention group, with significance level of alpha $=0.05 \%$ and $80 \%$ power, with an average follow-up of 2.04 years based on 24 months of recruitment and 36 months of maximum observation for outcomes, based on comparison between two Poisson rates, the needed sample size in each arm is 300. Loss to follow-up will not affect our ability to measure the primary outcome, as we are using administrative health data. We have extended our recruitment time from 24 months to 42 months, in response to slow recruitment at the beginning of the study.

\section{Allocation and blinding}

Participants will be randomly allocated after consent to either the patient navigator intervention or usual care in a 1:1 ratio using a computer-stratified generated randomisation sequence, generated a priori by a statistician (author ANA) with varying block sizes, stratified by primary clinic affiliation. Randomisation scheme will be executed in REDCap research software. ${ }^{21}$ Study coordinators at each site ascertain group allocation by clicking 'randomise' on REDCap, and inform participants of their assigned study arm.

Intervention assignment will not be blinded from trial participants, family members, research assistants or clinical teams. All patients/family participants will be blinded to the primary outcome (ER/urgent care visit) and hypothesised effects of the study. The navigators will not be blinded to the primary hypothesis. Full details of the navigator intervention will not be available to clinic staff/control participants to minimise contamination of the intervention to the control group. Data analysts will be blinded to group allocation and the nature of the intervention.

\section{Study intervention}

There will be one navigator in each of Calgary and Edmonton serving approximately 150 participants. These navigators are employees of Alberta Health Services (AHS), the organisation that provides governmentfunded healthcare to $>99 \%$ of Alberta residents. Individuals eligible for the patient navigator position will have a minimum of a Bachelor's degree in Social Work and 5 years of clinical experience, including experience working with adolescents and/or young adults. The navigator will be familiar with resources and health services available within AHS and the community. The intervention group will also receive usual care.

The intervention (personalised transition support, access to a patient navigator) is designed to overcome barriers and challenges experienced by transferring patients by facilitating a coordinated entry into the adult system, to increase appropriate use of adult-oriented ambulatory primary and specialty care, and reduce ER/ urgent care visits (primary outcome).${ }^{22}{ }^{23}$ We developed a structured navigator intervention with four distinct interrelated modules based on literature highlighting the need for each, ${ }^{5}$ our pretrial qualitative findings ${ }^{24}$ and in collaboration with content experts in transition models, partners in AHS and patient/parent advisory committees. We also developed a 2-day training programme for the navigators to complete prior to start of the trial. The training consists of readings, case scenarios and role plays. The modules are:

- Module 1, Prepare for transfer of care $^{25-28}$ : complete needs, risk and transition readiness assessments using a structured approach with modified SSHADESS psychosocial assessment ${ }^{29}$ (see online supplementary file 2), create medical passport, help establish relationships with primary care providers and appropriate specialty care providers, and enable timely attendance at first adult clinic visit.

- Module 2, Navigator as a health system broker ${ }^{30-32}$ : assist with data sharing between paediatric and adult service providers; work with patient and primary care providers to facilitate continuity of care; promote communication, collaboration and patient and family-centred care between all providers; and advocate with/for patient/family.

- Module 3, Social determinants of health $h^{33-35}$ : assist families with barriers related to social and economic determinants of health to reduce modifiable barriers to accessing ambulatory medical care after transfer.

- Module 4, Promote self-management of medical conditions $^{36-39}$ : provide information and access to tools, educational resources and peer support groups; track follow-up clinic visits, medication refills and laboratory testing in order to flag non-adherence early and provide coaching to reduce barriers to adherence; and plan for medical and/or mental health crisis management.

Once a participant is randomised to the intervention group, the navigators will contact the participant within 7 days to schedule a face-to-face (or phone meeting if necessary for rural dwelling patients) meeting during which the navigator will complete tasks in module 1. Using information obtained at this initial assessment, the navigators will use an adaptive, ${ }^{40}$ patient-centred approach that customises delivery of services based on needs of the patient, and consistent with principles and practice outlined in modules 2-4. Navigators will use telephone, 
text messages, email messages and in-person visits to maintain contact with participants as needed during the course of the intervention. Navigators will be alerted to ER/urgent care visits of participants by either the participants, caregivers (if appropriate), clinical providers or through use of electronic medical record alerts. The navigator will review circumstances related to ER/urgent care visits, and inform preventative actions based on the intervention modules. Scheduled patient reviews (in person or by telephone contact) will occur every 3 months (table 1 ). The navigators will record every contact and nature of assistance provided using standardised forms.

\section{Fidelity}

Procedures for monitoring adherence to intervention fidelity by the navigators will be managed by the Operational Oversight Committee (investigators, policy-makers, navigator's supervisors), and its role will be to assess and enhance fidelity to the intervention throughout the trial. The committee will review the patient navigator intervention using qualitative interviews of stakeholders and participants after the first five participants are enrolled into the intervention arm in each site. The knowledge gained from the review will be utilised to optimise the intervention protocol and address barriers to intervention fidelity across all sites.

Deviations will be carefully documented by navigators during their course of the trial. The navigators will complete a standardised fidelity checklist at the end of each patient encounter to assess their adherence to skills, interventions and pathways described in the intervention modules (see online supplementary file 3 ). Concomitant interventions which duplicate the intervention in whole or in part will be not be permitted during the trial.

\section{Usual care group}

Participants assigned to the usual care group will receive care as available within adult and paediatric clinics and the health region. However, this group is not a 'no intervention' group; in addition to care provided by their clinical teams, the study team will provide usual care participants with information in the form of infographics and quarterly newsletters, regarding transition to adult care resources such as young-adult-oriented transition websites, self-management tools and the opportunity to attend transition-focused workshops. Significant variation in transitional care is expected in this group within and across sites (based on our prior stakeholder engagement work).

To minimise attrition, all participants in the intervention and usual care group will receive electronic newsletters every 4 months, letters thanking them for their participation to date and email and phone reminders for follow-up data collection.

\section{Outcome and outcome measures}

Outcome measures and the assessment schedule are summarised in table 1 . The primary outcome is the rate of all-cause ER and urgent care visits during the observation period. Patients, providers and policy-makers on our team considered ER/urgent care visits to be relevant, and measureable in a blinded fashion across all clinical groups. We will obtain consent from trial participants to use their PHN to link with health service utilisation data. All ER and urgent care visits attributed to participants will be obtained from the National Ambulatory Care Reporting System, ${ }^{41}$ and the Clinical Analytics Team of AHS will conduct all analysis. AHS is the custodian of all Alberta Health data for $>99 \%$ of population.

We will evaluate ambulatory and inpatient care utilisation measures as secondary outcomes (primary care visits, specialty care ambulatory care visits, inpatient admissions, intensive care unit admissions and length of hospital stay). Outcome measure will be the rate of events. This data will be obtained from the Canadian Institute for Health Information Discharge Abstract Database ${ }^{42}$ and physician billing claims database. ${ }^{43}$

Other secondary outcomes are the Transition Readiness Assessment Questionnaire (TRAQ), and patient reported health status as measured by the 12-item Short Form Health Survey (SF-12). The TRAQ is the best-validated transition readiness scale for adolescents. ${ }^{44}{ }^{45}$ The questionnaire consists of 29 items, at grade 5.7 reading level and takes $\sim 5$ min to complete. Participants will complete the TRAQ online at baseline, 6, 12, 18 and 24 months. Regarding general health, participants will complete the 12-item SF-12 which is a validated 12-item survey that measures self-reported health status in individuals $>14$ years of age. ${ }^{46}$ The survey includes questions concerning physical functioning, role limitations because of physical health problems, bodily pain, general health perceptions, vitality (energy/fatigue), social functioning, role limitations because of emotional problems and general mental health (psychological distress and psychological wellbeing). Participants will complete the SF-12 at baseline, 6 , 12, 18 and 24 months.

We will prospectively capture the cost of the navigator intervention using microcosting methods ${ }^{47}$ (identification, measurement and valuation) that include one time and ongoing costs (development of materials, capital costs, wage rates for navigators, number of patients in caseload), enabling estimation of the cost of this intervention per patient served, using high quality administrative datasets from the AHS Clinical Analytics data repository. ${ }^{48}$

\section{Data monitoring and trial management}

The trial is governed by multiple stakeholder groups, including clinicians and policy-makers at each of the recruiting sites, study team members, and youth and family members. The Executive Trial Team is composed of the principal investigators and research team members, as well as site representatives and a patient representative. The team is supported by the larger Trial Management Committee, Operational Oversight Committee, Data Safety Monitoring Board (DSMB), Patient and Family 


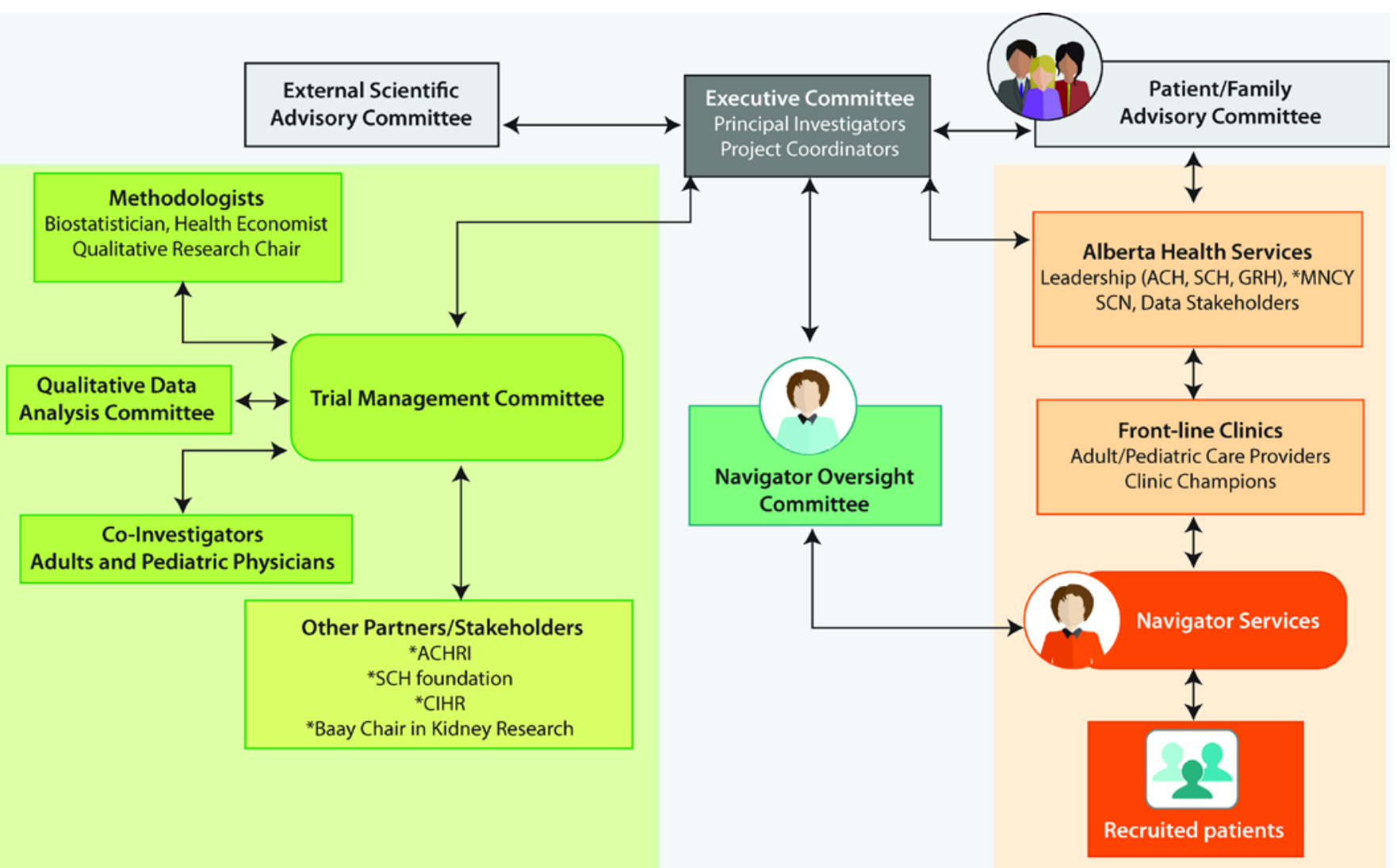

Figure 5 Governance of the Transition Navigator Trial. ACH, Alberta Children's Hospital; ACHRI, Alberta Children's Hospital Research Institute; GRH, Glenrose Rehabilitation Hospital; MNCY SCN, Maternal Newborn Child Youth Strategic Clinical Network; SCH, Stollery Children's Hospital.

Advisory Council and Scientific Advisory Board. A governance chart is provided in figure 5 .

Potential adverse events will be monitored in both study groups, however, the intervention is considered to be of minimal risk. No interim analysis is planned. The DSMB consists of three individuals who are familiar with the patient population and study question, but unfamiliar with the research team. The board will meet at least twice a year and monitor the trial in terms of safety of the participants and rigour of the data collection procedures.

\section{Analytical plan}

All analyses will be intention-to-treat. We will use Poisson regression to compare rates of ER/urgent care visits between the navigator and usual control groups, with fixed as well as random effects per site, and random effect by primary clinic. Demographic and medical characteristics that could be potential confounders or independent risk factors (eg, age, primary disease, socioeconomic status, location of residence, medical and mental health comorbidity in participant, ethnicity, immigrant status, demographic characteristics and medical/mental health of parents/caregivers obtained with consent) will be collected a priori, and used for adjusting the Poisson model. All other health utilisation outcomes will be analysed using descriptive statistical methods and by key demographic variables. For TRAQ and SF-12 scores, we will assess the effect of time (baseline, 6, 12, 18 and 24 months) on the scores using linear regression with random effects for subject and clinic.

For the economic evaluation, we will use established methods to enable comparisons of mean costs, as these are easily interpretable and relevant to healthcare payer. We will include the full cost of the navigator intervention (for intervention group) and the healthcare cost categories noted above and will use non-parametric bootstrap estimates to derive $95 \% \mathrm{CI}$ and mean cost differences between the treatment arms. ${ }^{49}{ }^{50}$ We will use 1000 biascorrected bootstrap replications (including sampling with replacement from the original data) to estimate the distribution of a sampling statistic to derive $95 \%$ CIs. ${ }^{49} \mathrm{We}$ will also compare cost by category (inpatient, ER, ambulatory care, physician claims) between both groups.

\section{Qualitative data analysis}

All interviews and focus groups will be audio-taped and transcribed verbatim, and NVivo software (QSR International, V.10, 2012) will be used for analysis. Thematic analysis will be used to extrapolate and systematically analyse patterns in the data generated by the qualitative interviews. ${ }^{50}$ We will closely adhere to the steps delineated by Braun and Clarke ${ }^{51}$ for conducting thematic analysis. We will use Krueger and Casey's ${ }^{52}$ constant comparative method of analysis to analyse the focus group data. This 
method involves 'cutting, sorting, and arranging through comparing and contrasting'. The coding process consists of grouping similar concepts and ideas, while identifying themes and categorising results. The research team will engage in established steps to increase the validity, credibility, transferability and dependability of findings by adhering to guidelines for publication of qualitative research studies. ${ }^{53}$

\section{Confidentiality}

The RCT and qualitative studies will adhere to the Personal Health Information Protection Act and all other regulatory and organisational standards for privacy, confidentiality and security of database information. All patient-identifiable electronic data will be stored in password-protected encrypted files on a secure network. Any identifiable information stored on REDCap will only be accessed by the investigative team and will be deidentified in the data export prior to analysis. All identifying information stored on paper will be stored in locked cabinets.

\section{Knowledge Translation}

We have used an integrated knowledge translation approach ${ }^{545}$ Our team is composed of patient representatives, researchers, clinical service providers and senior policy-makers who are committed to improving transition and transfer of care within Alberta. At the end of the study, we will conduct face to face stakeholder meetings to develop a holistic understanding of the barriers and facilitators to transitional care and the effectiveness of the patient navigator service using both quantitative and qualitative data obtained in this study.

\section{Patient and public involvement}

Our team is composed of patient representatives, researchers, clinical service providers and senior policymakers who are committed to improving transition and transfer of care within Alberta. We developed the intervention and strategy for implementation and evaluation after extensive consultation and engagement with stakeholders in subspecialty paediatrics and adult chronic disease clinics, emergency medicine, the Well on Your Way Transition Program at ACH, Calgary Zone Primary Care Networks, the Calgary Zone Primary Care \& Chronic Disease Management Program, the SCH Family-Centered Care Team and senior leadership within each tertiary care hospital. We conducted pretrial qualitative interviews and focus groups with relevant stakeholders (patients and families who recently transitioned to adult care, providers and policy-makers) to understand their perspectives regarding contextual variables affecting transition and refined the intervention based on results of this work. We engaged the Child and Youth Advisory Council (a patient council) at the Alberta Children's Hospital and through a ranking exercise we found that patients valued interventions with personal contact (eg, patient navigator, peer mentor support) more than those with less personal contact (social media, electronic apps). We are continuing to engage patient council groups such as these in Alberta. They will be informed of the results periodically during the study and also at the end of study through newsletters and news releases.

\section{DISCUSSION}

The TNT is a unique pragmatic population based trial which will address a significant gap in knowledge in the area of transition to adult care. The study will overcome previous methodological limitations including small sample sizes, non-generalisability due to diagnosisspecific inclusion criteria and non-randomised designs. The results will have the potential to change healthcare delivery, improve health outcomes and enhance experiences of young adults transitioning to adult care. The study will also provide a better understanding of the barriers and facilitators to transitional care and the effectiveness of the patient navigator service using both quantitative and qualitative data. We will determine what elements of the navigator service are most beneficial to participants, and whether the intervention is cost saving.

The evidence base for healthcare interventions during transition to adult care is limited by a paucity of data from controlled studies. Various interventions have been described and tested to a limited extent, mostly using non-randomised designs. Most are single centre, single disease studies, with limited generalisability. Gabriel et $a \bar{l}$ performed a systematic review of evidence focused on transition interventions. They report that structured transition interventions led to improvement in patient reported quality of life and perceived health status in several studies, suggesting potential publication bias. No studies have found significant cost savings; several studies found that having a structured transition process resulted in increased visits to the new adult provider, and a reduced time lag between the last paediatric visit and the first adult visitpstein ${ }^{56}$

The current study will be aligned with the Triple Aim Framework for health service evaluation. ${ }^{57}$ Interventions requiring highly skilled healthcare workers tend to be expensive, and to justify such an intervention, a cost evaluation is necessary. Complex interventions require assessment of fidelity to examine whether the intervention was delivered as intended, including a description of the interventions. This study will address these challenges.

In conclusion, this pragmatic RCT will evaluate the impact of a patient navigator service on rates of urgent care/ER visits and will provide patient, family and provider perceptions of the transition experience and the navigator service. This study will provide urgently needed data to guide paediatric and adult healthcare providers and policy-makers regarding optimal transitional care delivery. 
Author affiliations

${ }^{1}$ Section of Nephrology, Department of Pediatrics, University of Calgary, Calgary, Alberta, Canada

${ }^{2}$ Faculty of Social Work, University of Calgary, Calgary, Alberta, Canada

${ }^{3}$ Alberta Children's Hospital Research Institute, Department of Pediatrics, University of Calgary, Calgary, Alberta, Canada

${ }^{4}$ Division of Nephrology, Department of Medicine, University of Alberta, Edmonton, Alberta, Canada

${ }^{5}$ Department of Pediatrics, University of Calgary, Calgary, Alberta, Canada

${ }^{6}$ Section of Pediatric Oncology and Blood and Marrow Transplant, Alberta Children's Hospital, University of Calgary, Calgary, Alberta, Canada

${ }^{7}$ Section of Endocrinology, Department of Pediatrics, University of Calgary, Calgary, Alberta, Canada

${ }^{8}$ Section of Adolescent Medicine, Department of Pediatrics, University of Calgary, Calgary, Alberta, Canada

${ }^{9}$ Department of Emergency Medicine, University of Calgary, Calgary, Alberta, Canada ${ }^{10}$ Department of Pediatrics, University of Alberta, Edmonton, Alberta, Canada

${ }^{11}$ Department of Pediatrics, Autism Research Centre, University of Alberta,

Edmonton, Alberta, Canada

${ }^{12}$ Faculty of Nursing, University of Alberta, Edmonton, Alberta, Canada

${ }^{13}$ Department of Family Medicine, Department of Community Health Sciences, University of Calgary, Calgary, Alberta, Canada

${ }^{14}$ Section of Nephrology, Department of Pediatrics, University of Calgary, Calgary, Alberta, Canada

${ }^{15}$ Division of Cardiology, Department of Pediatrics, University of Alberta, Edmonton, Alberta, Canada

\section{Twitter Susan Samuel @drsamuelcalgary}

Acknowledgements We would like to acknowledge Sarah Gil for the creation of the figures used in this paper. We thank the patients and families who contributed to the development of this intervention and thedesign of the trial.

Collaborators Operational Oversight Committee: Catherine Morrison (Alberta Health Services), Jorge Pinzon (Department of Pediatrics, University of Calgary), Gail Andrew (Department of Pediatrics, University of Alberta), Catherine Hill (Alberta Health Services), Karen Johnston (Alberta Health Services), Susan Hughes (Alberta Health Services), Katie Byford Richardson (Alberta Health Services), Jane Bankes (Alberta Health Services Jake Jennings (Alberta Health Services), Kyleigh Schraeder (University of Calgary), Brooke Allemang (University of Calgary), Elizabeth Morgan-Maver (University of Calgary), Gurkeet Lalli (University of Calgary), Daniella San Martin-Feeney (University of Alberta), Laurel Ryan (University of Calgary). Trial Management Committee: Alberto Nettel-Aguirre (University of Calgary), Daniele Pacaud (Department of Pediatrics, University of Calgary), Greg Guilcher (Section of Pediatric Oncology and Blood and Marrow Transplant, University of Calgary), Eddy Lang (Department of Emergency Medicine, University of Calgary), Gail Andrew (Department of Pediatrics, University of Alberta), Iwona Wrobel (Department of Pediatrics, University of Calgary), Jorge Pinzon (Department of Pediatrics, University of Calgary), Kerry McBrien (Department of Family Medicine, Department of Community Health Sciences, University of Calgary), Lonnie Zwaigenbaum (Department of Pediatrics, Autism Research Centre, University of Alberta), Lorraine Hamiwka (Department of Pediatrics, University of Calgary), Scott Klarenbach (Department of Medicine, University of Alberta) April Elliott (Department of Pediatrics, University of Calgary), Catherine Morrison (Alberta Health Services), Catherine Hill (Alberta Health Services), Karen Johnston (Alberta Health Services), Kyleigh Schraeder (Department of Pediatrics, University of Calgary), Renee Farrell (Department of Pediatrics, University of Calgary), Elizabeth Morgan-Maver (University of Calgary), Gurkeet Lalli (University of Calgary), Daniella San Martin-Feeney (University of Alberta), Laurel Ryan (University of Calgary). Data Safety Monitoring Board: Dr. Mario Cappelli (Children's Hospital of Eastern Ontario Research Institute, University of Ottawa), Dr. Anne Stephenson (Institute of Health Policy, Management and Evaluation, University of Toronto), Dr. Mina Matsuda-Abedini (Division of Nephrology, The Hospital for Sick Children, University of Toronto). Scientific Advisory Board: Dr. Graham Reid (Children's Health Research Institute, University of Western Ontario), Dr. Bethany Foster (Research Institute of the McGill University Health Centre, McGill University), Dr. Astrid Guttmann (Senior Scientific Officer, Institute for Clinical Evaluative Sciences, University of Toronto)

Contributors AM, SS and GD conceived the idea for the study. SS, AM and GD wrote the first draft of the protocol. SS and KS wrote the first draft of the protocol manuscript and incorporated critical feedback from others. SK, ANA, GG, DP, JP, $\mathrm{EL}, \mathrm{GA}, \mathrm{LZ}, \mathrm{SS}, \mathrm{KM}$ and $\mathrm{LH}$ provided critical input into the development of the trial protocol and gave critical feedback on this manuscript. SS, AM and GD led the funding applications and all other authors contributed review of applications.

Funding Alberta Health Services (Research Number 1040209), Alberta Children's Hospital Foundation (Research Number 1042146), Stollery Children's Hospital Foundation, Canadian Institutes of Health Research (388256-CHI-CBBA-161557).

Competing interests None declared.

Patient consent for publication Not required.

Ethics approval Ethics approval was obtained from the University of Calgary Conjoint Health Research Ethics Board (REB \#162561) and the University of Alberta Health Research Ethics Board (Pro00077325).

Provenance and peer review Not commissioned; peer reviewed for ethical and funding approval prior to submission.

Open access This is an open access article distributed in accordance with the Creative Commons Attribution Non Commercial (CC BY-NC 4.0) license, which permits others to distribute, remix, adapt, build upon this work non-commercially, and license their derivative works on different terms, provided the original work is properly cited, appropriate credit is given, any changes made indicated, and the use is non-commercial. See: http://creativecommons.org/licenses/by-nc/4.0/.

\section{ORCID iDs}

Susan Samuel http://orcid.org/0000-0003-2224-448X

Kyleigh Schraeder http://orcid.org/0000-0002-5000-3264

\section{REFERENCES}

1 Kaufman M, Pinzon J, Adolescent Health Committee. Transition to adult care for youth with special health care needs. Paediatr Child Health 2007;12:785-8.

2 Bloom SR, Kuhlthau K, Van Cleave J, et al. Health care transition for youth with special health care needs. J Adolesc Health 2012:51:213-9.

3 Van Walleghem N, MacDonald CA, Dean HJ. Evaluation of a systems navigator model for transition from pediatric to adult care for young adults with type 1 diabetes. Diabetes Care 2008;31:1529-30.10.2337/dc07-2247

4 Prestidge C, Romann A, Djurdjev O, et al. Utility and cost of a renal transplant transition clinic. Pediatric Nephrology 2012;27:295-302.

5 Gabriel P, McManus M, Rogers K, et al. Outcome evidence for structured pediatric to adult health care transition interventions: a systematic review. J Pediatr 2017;188:263-9.

6 Chan A-W, Tetzlaff JM, Altman DG, et al. Spirit 2013: new guidance for content of clinical trial protocols. The Lancet 2013;381:91-2.

7 Chan A-W, Tetzlaff JM, Altman DG, et al. Spirit 2013 statement: defining standard protocol items for clinical trials. Ann Intern Med 2013;158:200-7.

8 Chan A-W, Tetzlaff JM, Gøtzsche PC, et al. Spirit 2013 explanation and elaboration: guidance for protocols of clinical trials. BMJ 2013;346:e7586.

9 Zwarenstein M, Treweek S, Gagnier JJ, et al. Improving the reporting of pragmatic trials: an extension of the CONSORT statement. BMJ 2008;337:a2390.

10 Tong A, Sainsbury P, Craig J. Consolidated criteria for reporting qualitative research (COREQ): a 32-item checklist for interviews and focus groups. Int J Qual Health Care 2007;19:349-57.

11 Network TE. Enhancing the quality and transparency of health research. Available: https://www.equator-network.org

12 Bindels-de Heus KGCB, van Staa A, van Vliet I, et al. Transferring young people with profound intellectual and multiple disabilities from pediatric to adult medical care: parents' experiences and recommendations. Intellect Dev Disabil 2013;51:176-89.

13 Foster BJ, Dahhou M, Zhang X, et al. Association between age and graft failure rates in young kidney transplant recipients. Transplantation 2011;92:1237-43.

14 Samuel SM, Nettel-Aguirre A, Hemmelgarn BR, et al. Graft failure and adaptation period to adult healthcare centers in pediatric renal transplant patients. Transplantation 2011:91:1380-5.

15 Yeung E, Kay J, Roosevelt GE, et al. Lapse of care as a predictor for morbidity in adults with congenital heart disease. Int J Cardiol 2008;125:62-5

16 Cohen E, Gandhi S, Toulany A, et al. Health care use during transfer to adult care among youth with chronic conditions. Pediatrics 2016;137:e20152734.

17 Sevick MA, Trauth JM, Ling BS, et al. Patients with complex chronic diseases: perspectives on supporting self-management. J Gen Intern Med 2007;22:438-44. 
18 Mackie AS, Islam S, Magill-Evans J, et al. Healthcare transition for youth with heart disease: a clinical trial. Heart 2014;100:1113-8.

19 Mackie AS, Rempel GR, Kovacs AH, et al. A cluster randomized trial of a transition intervention for adolescents with congenital heart disease: rationale and design of the Chapter 2 study. BMC Cardiovasc Disord 2016;16:127

20 Holmes-Walker DJ, Llewellyn AC, Farrell K. A transition care programme which improves diabetes control and reduces hospital admission rates in young adults with type 1 diabetes aged 15-25 years. Diabet Med 2007;24:764-9.

21 Harris PA, Taylor R, Thielke R, et al. Research electronic data capture (REDCap) - A metadata-driven methodology and workflow process for providing translational research informatics support. J Biomed Inform 2009;42:377-81.

22 Betz CL, Redcay G. Dimensions of the transition service coordinator role. J Spec Pediatr Nurs 2005;10:49-59.

23 Betz CL, Redcay G. Lessons learned from providing transition services to adolescents with special health care needs. Issues Compr Pediatr Nurs 2002;25:129-49.

24 Dimitropoulos G, Morgan-Maver E, Allemang B, et al. Health care stakeholder perspectives regarding the role of a patient navigator during transition to adult care. BMC Health Serv Res 2019;19. Accepted June 6, 2019

25 Cooley WC, Sagerman PJ, American Academy of Pediatrics. Supporting the health care transition from adolescence to adulthood in the medical home. Pediatrics 2011;128:182-200.

26 van Staa A, van der Stege HA, Jedeloo S, et al. Readiness to transfer to adult care of adolescents with chronic conditions: exploration of associated factors. J Adolesc Health 2011;48:295-302.

27 McManus M, White P. Transition to adult health care services for young adults with chronic medical illness and psychiatric comorbidity. Child Adolesc Psychiatr Clin N Am 2017;26:367-80.

28 BC Children's Hospital. Transition to adult care. Available: http:// www.bcchildrens.ca/Services/SpecializedPediatrics/YouthHealth/ Resources.htm

29 American Academy of Pediatrics, Kinsman SB, Ginsburg KR, eds. Reaching Teens Strength-Based Communication Strategies To Build Resilience and Support Healthy Adolescent Development. American Academy of Pediatrics, 2014: 635.

30 Coyne B, Hallowell SC, Thompson M. Measurable outcomes after transfer from pediatric to adult providers in youth with chronic illness. J Adolesc Health 2017;60:3-16.

31 Suris J-C, Akré C, Rutishauser C. How adult specialists deal with the principles of a successful transition. J Adolesc Health 2009;45:551-5.

32 Lipstein EA, Muething KA, Dodds CM, et al. "I'm the One Taking It": Adolescent Participation in Chronic Disease Treatment Decisions. J Adolescent Health 2013;53:253-9.

33 Ravani P, Bonanni A, Rossi R, et al. Anti-Cd20 antibodies for idiopathic nephrotic syndrome in children. Clin J Am Soc Nephrol 2016;11:710-20.

34 Cramm JM, Strating MMH, Sonneveld HM, et al. The longitudinal relationship between satisfaction with transitional care and social and emotional quality of life among chronically ill adolescents. Appl Res Qual Life 2013;8:481-91.

35 Grant C, Pan J. A comparison of five transition programmes for youth with chronic illness in Canada. Child: Care, Health and Development 2011;37:815-20.

36 Williams T, Sherman EMS, Mah JK, et al. Measurement of medical self-management and transition readiness among Canadian adolescents with special health care needs. Int J Child Adolescent Health 2010;3:527-35.

37 Akre C, Suris J-C. From controlling to letting go: what are the psychosocial needs of parents of adolescents with a chronic illness? Health Educ Res 2014;29:764-72.

38 McGrady ME, Hommel KA. Medication adherence and health care utilization in pediatric chronic illness: a systematic review. Pediatrics 2013;132:730-40.

39 Maslow GR, Chung RJ. Systematic review of positive youth development programs for adolescents with chronic illness. Pediatrics 2013;131:e1605-18.

40 Almirall D, Chronis-Tuscano A. Adaptive interventions in child and adolescent mental health. J Clin Child Adolesc Psychol 2016;45:383-95.

41 Canadian Institute for Health Information. National ambulatory care reporting system metadata. Available: https://www.cihi.ca/en/ national-ambulatory-care-reporting-system-metadata

42 Canadian Institute for Health Information. Discharge Abstract database. data elements 2018-2019. Available: https://www.cihi.ca/ sites/default/files/document/dad-data-elements-2018-en-web.pdf

43 Canadian Institute for Health Information. National physician database data release, 2015-2016: methodological notes. Ottawa, ON: CIHI, 2017.

44 Sawicki GS, Lukens-Bull K, Yin X, et al. Measuring the transition readiness of youth with special healthcare needs: validation of the TRAQ - Transition readiness assessment questionnaire. J Pediatr Psychol 2011;36:160-71.

45 Zhang LF, Ho JSW, Kennedy SE, et al. A systematic review of the psychometric properties of transition readiness assessment tools in adolescents with chronic disease. BMC Pediatr 2014;14:4 http:// www.biomedcentral.com/1471-2431/14/4

46 Ware J, Kosinski M, Keller SD. A 12-Item short-form health survey: construction of scales and preliminary tests of reliability and validity. Med Care 1996;34:220-33.

47 Gold MR, Siegel J, Russell LB, et al. Cost Effectiveness in Health and Medicine. New York: Oxford University Press, 1996.

48 McBrien KA, Manns BJ, Chui B, et al. Health care costs in people with diabetes and their association with glycemic control and kidney function. Diabetes Care 2013;36:1172-80.

49 Desgagné A, Castilloux AM, Angers JF, et al. The use of the bootstrap statistical method for the pharmacoeconomic cost analysis of skewed data. Pharmacoeconomics 1998;13:487-97.

50 Davison AC, Hinkley DV. Bootstrap methods and their application. Vol 1. Cambridge university press, 1997.

51 Braun V, Clarke V. Using thematic analysis in psychology. Qual Res Psychol 2006;3:77-101.

52 Krueger RAaC MA. Focus groups: A Practical Guide for Applied Research. 5 ed. Sage Publications, 2014.

53 Elliott R, Fischer CT, Rennie DL. Evolving guidelines for publication of qualitative research studies in psychology and related fields. $\mathrm{Br} \mathrm{J}$ Clin Psychol 1999;38:215-29.

54 Graham ID, Logan J, Harrison MB, et al. Lost in knowledge translation: time for a MAP? J Contin Educ Health Prof 2006;26:13-24.

55 Straus SE, Tetroe J, Graham I. Defining knowledge translation. Can Med Assoc J 2009;181:165-8.

56 Mackie AS, Rempel GR, Kovacs AH, et al. Transition intervention for adolescents with congenital heart disease. J Am Coll Cardiol 2018;71:1768-77.

57 Berwick DM, Nolan TW, Whittington J. The triple aim: care, health, and cost. Health Aff 2008;27:759-69. 